\title{
In vivo evaluation of bioresorbable polylactide implants for cervical graft containment in an ovine spinal fusion model
}

\author{
G. Bryan Cornwall, Ph.D., P.Eng., Christopher P. Ames, M.D., \\ Neil R. Crawford, Ph.D., Robert H. Chamberlain, M.S., Anthony M. Rubino, \\ Howard B. Seim III, D.V.M., AND A. Simon Turner, B.V.Sc., M.S.
}

\author{
MacroPore Biosurgery, Incorporated, San Diego, California; Department of Neurological Surgery, \\ University of California, San Francisco, California; Spinal Biomechanics Research Laboratory, \\ Barrow Neurological Institute, Phoenix, Arizona; and Department of Clinical Sciences, \\ Colorado State University, Fort Collins, Colorado
}

\begin{abstract}
Object. An in vivo study was conducted in an ovine model to investigate the biomechanical changes after the animals underwent single-level anterior cervical discectomy followed by fusion in which autologous tricortical graft was used and implantation of cervical plates for which bioresorbable polymer screws and plates were used. The specific aims of the study were to evaluate whether implant failure or screw backout would occur over time and to measure the change in stiffness at the treated level at various postoperative time periods (3, 6, and 12 months).

Methods. A total of 58 x-ray films were evaluated over the 12-month survival period. No screw breakage or displacement was observed in any animal during the temporal radiographic analysis. Radiographically confirmed fusion appeared to be complete at all time periods longer than 6 months. The biomechanical testing demonstrated dramatic reductions in range of motion at the fusion level in the animals allowed to survive for 6 and 12 months, indicating complete fusion after 6 months.

Conclusions. The bioresorbable polymer cervical graft containment system appears to provide a safe and effective alternative for cervical fusion, and warrants further clinical evaluation for its use in single-level anterior cervical discectomy and fusion without postoperative orthosis.
\end{abstract}

\section{KEY WORDS - cervical spine • bioresorbable polymer • biomechanical study • animal model • sheep}

The use of anterior cervical plates in spinal fusion surgery is increasing. In several biomechanical studies investigators have demonstrated the added stability provided by these plates, which theoretically may result in increased fusion rates. ${ }^{3,12,21}$ Although fusion rates are high in uninstrumented anterior cervical fusions, high morbidity rates have been reported in connection with the use of autologous bone, ${ }^{5,26}$ and the possibility of graft extrusion often requires the patient to wear a rigid cervical orthotic device. ${ }^{23,26}$ Furthermore, in two-level anterior cervical discectomies, the pseudarthrosis rates are significantly higher in patients treated without plate fixation. ${ }^{25}$

The advantages of anterior cervical screw plates include their mechanical effectiveness for providing internal fixation, their ability to prevent graft extrusion or displacement, to maintain compression of graft materials, and to reduce the reliance on external cervical orthoses postoperatively. $3,12,25$ The disadvantages of cervical screw plates include numerous potential complications (that is, hardware failure, subsidence, loss of deformity correction, and/or dysphagia). ${ }^{18,24}$ These effects are thought to be decreased with dynamic variable-angle screw plates, which

Abbreviations used in this paper: $\mathrm{LZ}=$ lax zone; $\mathrm{ROM}=$ range of motion; $\mathrm{SZ}=$ stiff zone. allow for greater load sharing in the setting of normal subsidence. ${ }^{3,21}$ The complication rates are lower for modern fusions in which plates are used, and these procedures have reduced bone graft donor-site morbidity. ${ }^{26}$

Bioresorbable polymer implants fabricated commercially (MacroPore Biosurgery, Inc., San Diego, CA) have been used clinically for many years in neurosurgical applications such as cranial base reconstruction, ${ }^{16}$ and in more load-bearing applications such as distraction osteogenesis in craniofacial reconstructions. ${ }^{7}$ The material resorbs by hydrolysis within 12 to 18 months, gradually decreasing in strength after 6 months. ${ }^{13,19}$ It is possible to increase the thickness of this mesh to dimensions that may provide sufficient strength for a graft containment function in the cervical spine. ${ }^{1}$ Previously reported clinical experience has indicated that bioresorbable polymer implant systems could be effective for anterior cervical discectomy and fusion in terms of preventing graft migration and providing interim stability while fusion is progressing. ${ }^{24}$

Authors of various studies have reported using animal models for in vivo or in vitro evaluation of the cervical spine. The goat model ${ }^{2,20,22}$ and the sheep model ${ }^{4,14,15,17,27,28}$ appear to be the most prevalent. Kandziora, et al., ${ }^{15}$ provided a detailed comparison of human and sheep cervical spines, including anatomical, radiographic, bone mineral 
density, and biomechanical evaluations. They concluded from their collected quantitative data that the C3-4 motion segment in sheep appeared to be the most reliable model to compare with the human cervical spine.

We previously reported a pilot study of the biomechanical effectiveness of a prototype graft containment plate and screw system fabricated with a bioresorbable polymer. ${ }^{1}$ To understand better how the biomechanics change after bone healing and plate resorption, we used an ovine model in which a single-level anterior cervical discectomy was performed and fusion was accomplished using an autograft consisting of tricortical iliac crest graft and bioresorbable plates and screws. Normal intact cervical spines were compared with specimens recovered after discectomy, grafting, and ventral (anterior) plate implantation at time intervals immediately after and at 3, 6, and 12 months after surgery. Our study's specific aims were to evaluate whether the implants would fail or screws would back out over time, and to measure the change in stiffness at the treated level at different postoperative time periods.

\section{MATERIALS AND METHODS}

\section{In Vivo Study}

Thirteen adult, skeletally mature female Rambouillet $\mathrm{X}$ Columbian ewes were used in the study. Approval for the surgeries performed in these sheep was granted by the Colorado State University Animal Care and Use Committee. Colorado State University complies with the US Department of Agriculture regulations promulgated under the authority of the Animal Welfare Act and those of the US Public Health Service Policy on Laboratory Animal Care as provided in the Health Research Extension Act. In addition, the program complies with the recommendations of the American College of Laboratory Animal Medicine and the "Guide for the Care and Use of Laboratory Animals."

The biomechanical stability of the developing cervical fusion was evaluated postoperatively and after 3, 6, and 12 months in vivo (two animals per follow-up period). Four specimens were used as samples of healthy sheep spine. Two samples were assessed in the immediate postoperative group. Radiographic assessment of the implants was performed in the eight animals immediately postoperatively, at 1-month intervals for the first 6 months, and at 3month intervals for the remainder of the survival period. Both frontal and sagittal plane x-ray films were obtained at each time point.

\section{Resorbable Polymer Implants}

The resorbable polymer implants were fabricated using an amorphous copolymer of 70:30 poly(L-lactide-co-D,Llactide), which was sterilized using e-beam irradiation (MacroPore Biosurgery, Inc.). The plate was $18 \mathrm{~mm}$ wide by $26 \mathrm{~mm}$ long and $1.6 \mathrm{~mm}$ thick. The screws were 2.7 $\mathrm{mm}$ in diameter by $12 \mathrm{~mm}$ in length. Two screws were used in each level of the single-level fusion construct; four screws total were used in each animal. Every screw had two 1-mm-diameter tantalum beads (RSA Biomedical, Umea, Sweden), one placed at the head and the other at the tip to facilitate radiographic monitoring of the implant positions. Each plate had two 0.5-mm-diameter tantalum beads placed at both the cranial and caudal ends to distinguish the position of the plate from that of the screws.

\section{Surgical Procedure}

Animals were denied food for 24 hours before surgery, but water intake was not restricted during this time. Anesthesia was induced with ketalar $(4 \mathrm{mg} / \mathrm{kg}$ ) and diazepam (7.5 mg total). After induction of anesthesia, sheep were maintained on isoflurane (1.5-3\%) in $100 \% \mathrm{O}_{2}$ at approximately $2 \mathrm{~L} /$ minute during the surgical procedure. Muscle relaxants were not used.

The sheep were first placed in a lateral position to expose the left iliac crest for removal of a tricortical autograft. The animals were then repositioned for a ventral (anterior) exposure of the $\mathrm{C} 3-4$ disc. $^{15}$ The disc material was removed and the disc space was distracted. Endplates were prepared using a high-speed burr (Medtronic Midas Rex, Fort Worth, TX). The tricortical graft was cut to size and placed in the distracted disc space, the bioresorbable polymer plate was heated in a sterile saline hot water bath $\left(\sim 65^{\circ} \mathrm{C}\right)$ above the glass transition temperature of the polymer $\left(\sim 55^{\circ} \mathrm{C}\right)$ and contoured to the ventral anatomy of the sheep cervical spine to cover the autograft, as shown in Fig. 1. Two 2.7-mm-diameter screws were placed at each level (four screws in all) by using a drill/tap to prepare the hole before insertion of the bioresorbable polymer screw. As previously described, ${ }^{1}$ the hole size was designed to accommodate the minor diameter of the screw. When tapping the bone, the tap also created a thread in the bioresorbable plate and thus provided an interference fit that helped create a locking mechanism to prevent screw backout. The soft tissues were closed in a routine manner and the animals were allowed to ambulate soon after recovery from anesthesia.

Pain medication was administered after the surgical procedures; this included fentanyl patches at a dose of $150 \mu \mathrm{g} / \mathrm{hour}$ administered with a continuous percutaneous patch for 3 days. Additional pain medication included $1 \mathrm{~g}$ phenylbutazone administered orally once daily for 3 days. At the conclusion of the randomly assigned survival period, all sheep were humanely killed with a barbiturate overdose.

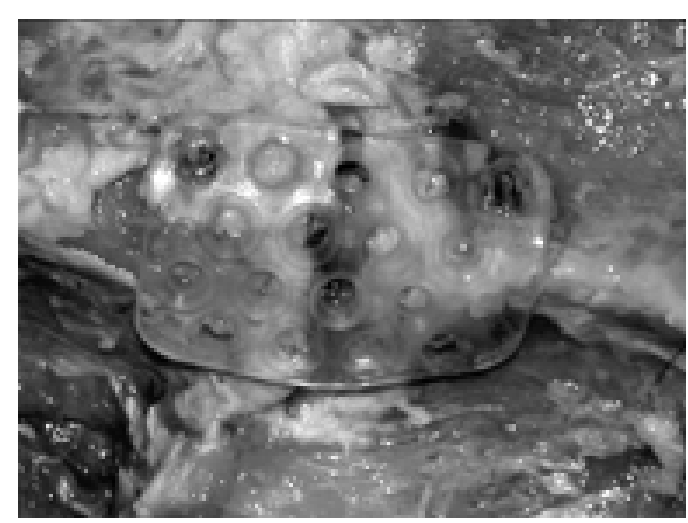

Fig. 1. Photograph showing placement of bioresorbable graft containment plate on cervical sheep spine. 


\section{Biomechanical Testing}

The entire cervical spine was extracted to prepare for biomechanical analysis. In most specimens, the C3-4 segment was the level at which an obvious fusion mass or plate was attached. The fused level plus one adjacent cranial and one caudal vertebra were isolated (four-vertebra segments); any additional levels were dissected and discarded. Specimens were "potted" for testing by inserting three household wood screws in the exposed distal endplates and joint articulations and embedding the heads of these screws and a portion of each vertebra in polymethyl methacrylate poured into cylindrical aluminum fixtures. The caudal fixture was rigidly attached to the base of the testing apparatus and loads were applied to the cranial fixture. A clear fusion mass was found to be present in the 3-, 6-, and 12-month animals. This mass was left undisturbed during preparation of specimens for testing.

Each tested specimen consisted of the treated motion segment and the adjacent cranial and caudal motion segments. For flexibility testing, torque loading was applied to each specimen through a system of cables and pulleys in conjunction with a standard servohydraulic test system (MTS, Minneapolis, MN), as we have described previously. ${ }^{8}$ Loads were applied to induce flexion/extension, lateral bending, and axial rotation. Three cycles of preconditioning (ramp from 0-5 Nm, 60-second hold, ramp from 5-0 Nm) were applied before data were recorded. The maximum load of $5 \mathrm{Nm}$ exceeds the $1.5 \mathrm{Nm}$ typically used in testing the human cervical spine ${ }^{6}$ because the sheep spines were larger and more rigid. After allowing the specimen to rest for 60 seconds at zero load, specimens were loaded quasistatically to a maximum of 5 $\mathrm{Nm}$ in 1-Nm increments. Each increment was held for 45 seconds.

Three-dimensional specimen motion in response to the loads was determined using the Optotrak 3020 system
(Northern Digital, Waterloo, ON, Canada). This system records the motion of three infrared-emitting diodes per vertebra that are mounted to the ends of surgical guidewires rigidly inserted into each vertebra. Custom software was used to convert the marker coordinates to angles around each of the anatomical axes..$^{9,11}$ From the quasistatic load-deformation data, the angular LZ, SZ, and ROM were quantified as measures of motion segment stability. ${ }^{10}$ The ROM is the angle achieved at maximum load and the $\mathrm{LZ}$ and SZ are the portions of the ROM before and after there is substantial ligamentous resistance. No statistical analysis was possible for these data because most groups were composed of only two specimens.

\section{RESULTS}

There were no surgical or postoperative complications in any animal. All sheep were neurologically normal and without ataxia at the time of death. A total of 58 lateral radiographs were evaluated over the 12-month survival period; a summary of the X-ray films obtained at the survival times of 3, 6, and 12 months is shown in Figs. 2 through 5.

There was no obvious screw breakage or displacement observed in any animal during the temporal radiographic analysis at any time period: not between the postoperative and 3-month follow-up evaluations (summarized in Fig. 3 ), between the 3- and 6-month follow up (summarized in Fig. 4), or between the 6- and 12-month follow up (summarized in Fig. 5). Radiographically confirmed fusion appeared to be complete at all time points later than 6 months.

The biomechanical testing results are summarized in Fig. 6. The data for the entire spine segment, including treated and adjacent levels, is summarized in Fig. 6A. The $\mathrm{ROM}$ at the fusion level is summarized in Fig. 6B.

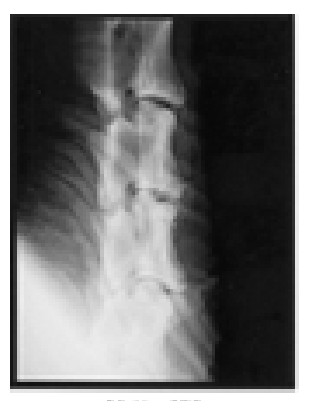

$\infty 0 \mathrm{Mo}-275$

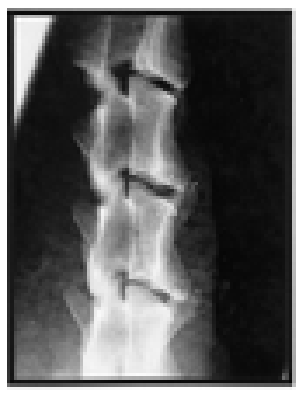

Q6 Ma-25T

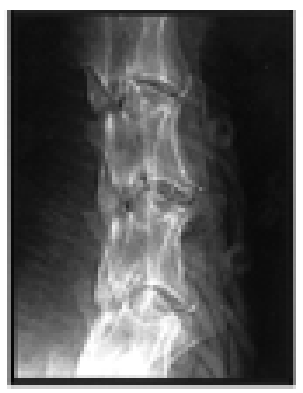

00 Mo-20ns

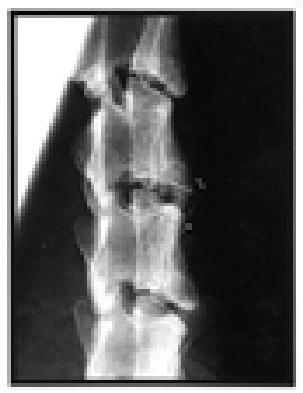

60 me-2te

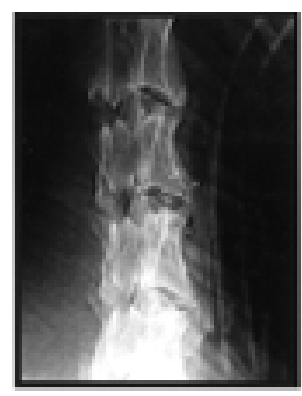

$\infty$ M.o. $2 n$

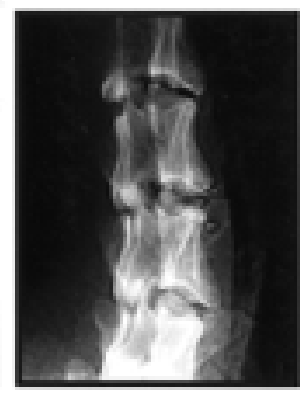

$\infty$ Mo-241

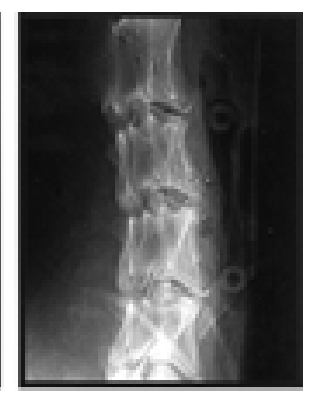

60 Na-2ms

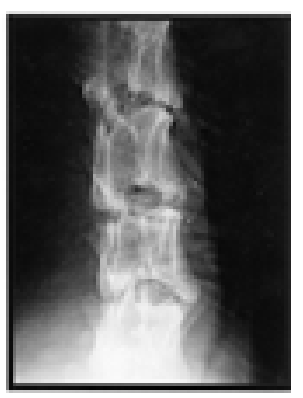

go ke-2ar

Fig. 2. Radiographs obtained immediately postoperatively in eight sheep treated with bioresorbable implants. 
G. B. Cornwall, et al.

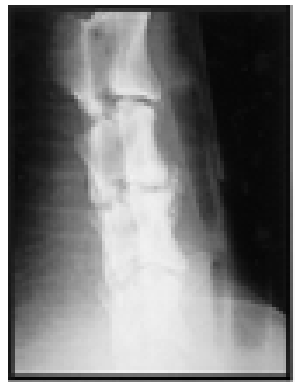

es Mo-2M

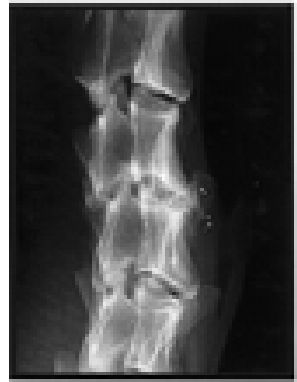

64 Ma-2W

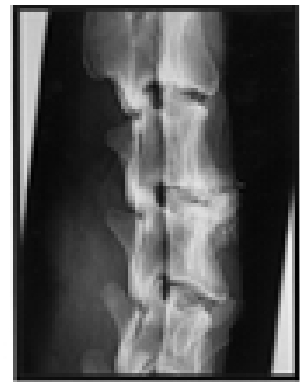

64 Mo-276

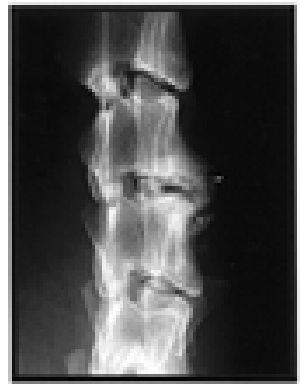

99 Merats

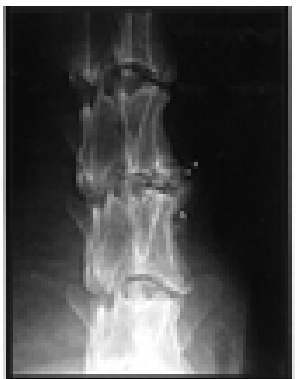

Q9 Morar

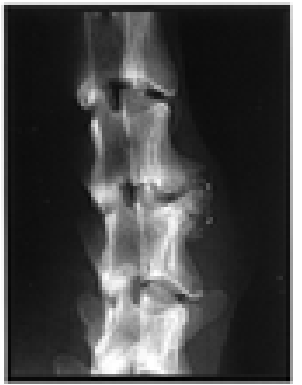

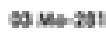

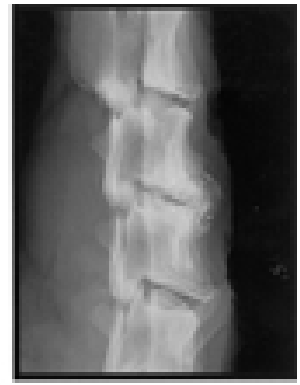

Q9 40,23

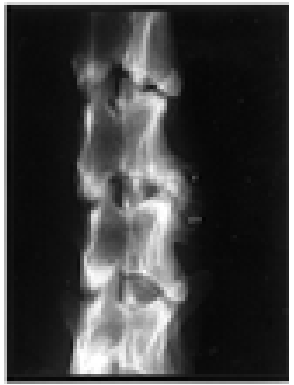

Q9 Mar-219

Fig. 3. Radiographs obtained 3 months postoperatively in eight sheep treated with bioresorbable implants.

In the two animals allowed to survive for 3 months, there was slight reduction in motion at the fusion level compared with the adjacent cranial and caudal levels. In these animals, the ROM at the fusion level was composed of $65 \% \mathrm{LZ}$ and 35\% SZ. Dramatic reductions in LZ, SZ, and ROM to $0.28,0.86$, and $0.97^{\circ}$ or less, respectively, were seen at the fusion level in 6-and 12-month animals. In the 6- and 12-month animals, the ROM was composed of $13 \% \mathrm{LZ}$ and $87 \% \mathrm{SZ}$ at the fusion level. The ROM at the adjacent levels remained relatively constant regardless of the condition at the fusion level. On average, the ROM at adjacent levels remained composed of $72 \% \mathrm{LZ}$ and $28 \% \mathrm{SZ}$.

\section{DISCUSSION}

The ability to heat the bioresorbable polymer plate above the glass transition temperature and to contour the

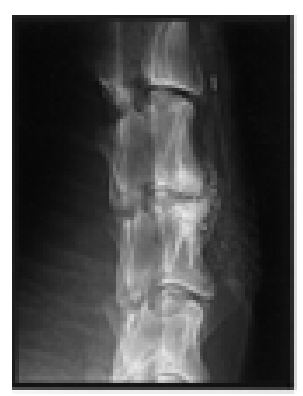

64 Ma-2)s

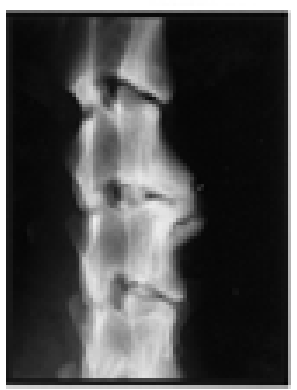

O4 Ho-2as

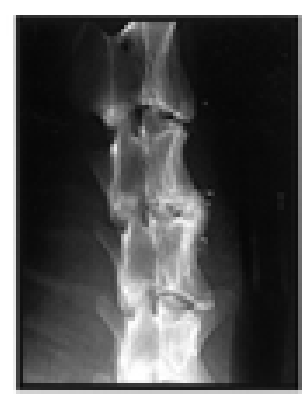

OS Mo-2m

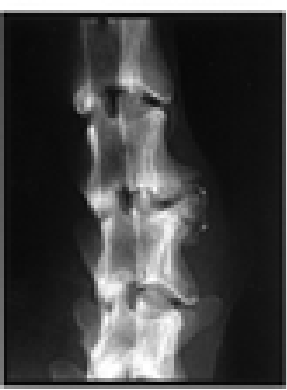

Q4 Ma-2a1

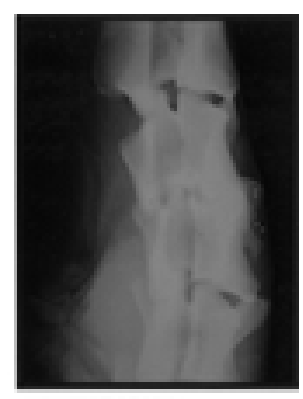

O4 $100-2 n$

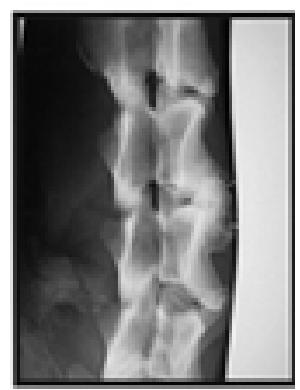

of Mo-ata

Fig. 4. Radiographs obtained 6 months postoperatively in six sheep treated with bioresorbable implants. 


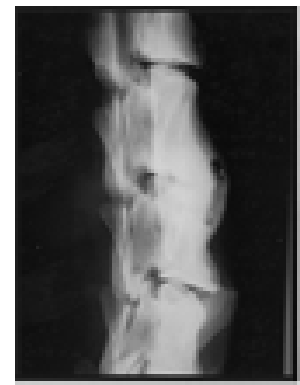

12 Me-27s

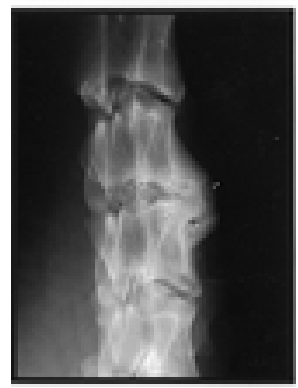

12 Mo-2as

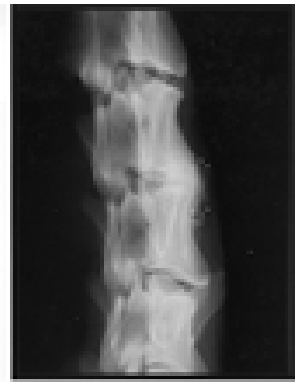

$12 \operatorname{man}-2 m$

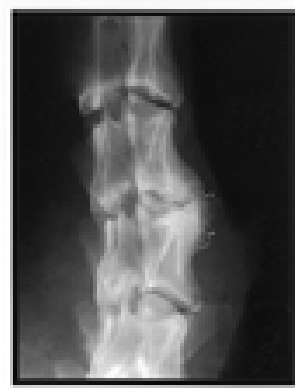

12 Magall
Fig. 5. Radiographs obtained 12 months postoperatively in four sheep treated with bioresorbable implants.

plate to the ventral (anterior) surface of the sheep cervical spine helped to provide a three-dimensional construct that matched the native anatomy. This ability easily to contour the implants has been successfully used clinically. ${ }^{7,24}$

The radiographic assessment indicated that there was no displacement of the graft material, and no implant failure or screw backout was observed on any radiograph obtained at any time point in all eight animals.

The biomechanical evaluation demonstrated a significant reduction in motion in all three planes (flexion/extension, lateral bending, and axial rotation) at the 6- and 12month survival periods. Although reduction in motion can be achieved by fixation without fusion, the very small absolute value to which motion was reduced after 6 months $\left(<1^{\circ}\right)$ indicates complete bone fusion. Maintenance of a constant level of motion at the adjacent segments regardless of the condition of the treated spine supports the hypothesis that the reduced motion at the treated level was due to bone fusion and not to generalized postsurgical stiffening.

In addition to studying the ROM, we quantified the component LZ and SZ values. Although they are not necessary to demonstrate fusion, these parameters provide additional information on the biomechanics at the fused and adjacent levels. The finding that the fused segment exhibited primarily an SZ (87\%) with very little LZ (13\%) indicates that most of the motion probably occurred because of the elastic bending of bone without the lax region of bending that would exist in joint interfaces separated by a gap (that is, when fusion is not present). The finding that the ratios of LZ and SZ to total ROM remained relatively constant at adjacent levels is confirmation that not just the total deformation but also the nature of the load-deformation response at adjacent levels was unchanged and that adjacent levels were relatively unaffected by the surgery.
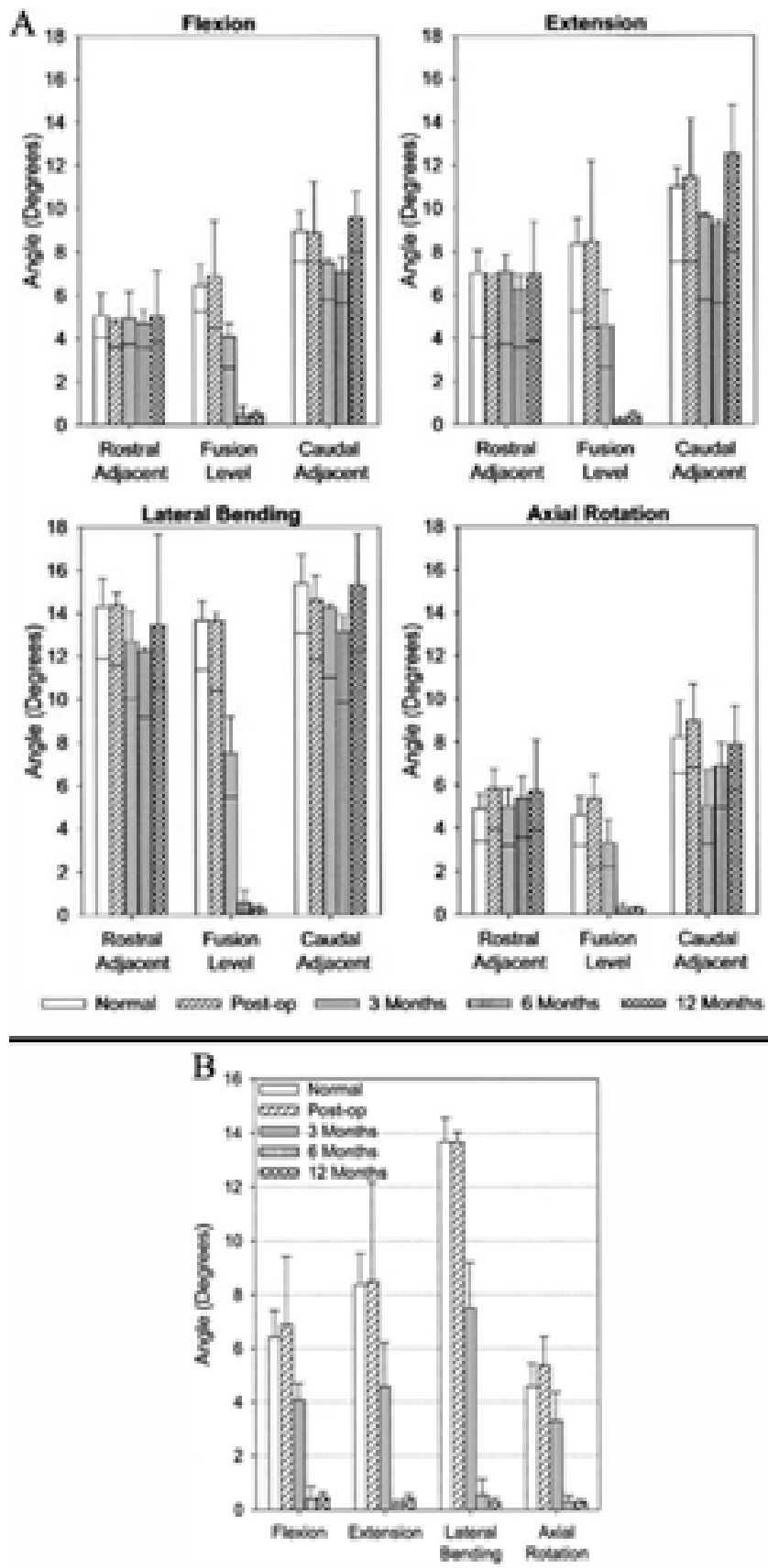

Fig. 6. Bar graphs showing biomechanical results in sheep treated with bioresorbable implants. A: The mean angular motion at the level of fusion and adjacent levels during each loading mode studied. The horizontal line on each column denotes the division between LZ and SZ. The entire column represents the ROM. Error bars show standard deviation of the ROM. B: Summary of the mean angular ROM in all planes at the fusion level. Error bars show the standard deviation.

No nonplate-treated control sheep were used in the in vivo evaluation because previous evaluations have led to graft extrusion and neurological compromise of the animals. Too many animals in these control groups were killed early and the Animal Care and Use Committee would not provide approval for additional nonplate-treat- 
ed control animals. The sheep model is an aggressive one for the in vivo evaluation of anterior cervical spine instrumentation because of the amount of motion that occurs soon after surgery.

A limitation of this research is the relatively small number of specimens studied per group; however, the plate performed as hoped in this study, containing the graft without evidence of screw backout or migration. In all six animals that were allowed to survive longer than 3 months postimplantation, the treated area progressed to eventual solid fusion. Positive clinical experience has also been reported in studies in which a similar bioresorbable plating construct is used. ${ }^{24}$ The authors reported that the construct was shown to be effective in preventing graft migration and providing interim stability during the fusion consolidation period. Previous biomechanical evaluation ${ }^{1}$ in which human cadavers were used demonstrated that the construct acted most effectively as a tension band.

Other cervical biomechanical studies have demonstrated the theoretical advantages of dynamic cervical plating systems. ${ }^{3}$ The bioresorbable polymer implant construct may have acted as a dynamic plate due to the flexibility and compliance of the polymer material properties. The surgeon should not expect the bioresorbable polymer plate to provide the same stabilizing effect as a metal one, but can consider it to be an excellent alternative if a graft containment device is needed that also provides limited resistance to motion, especially resistance to extension.

The bioresorbable cervical graft containment system appears to provide a safe and effective alternative for cervical fusion and warrants further clinical evaluation for single-level anterior cervical discectomy without postoperative orthosis.

\section{References}

1. Ames CP, Cornwall GB, Crawford NR, et al: Feasibility of a resorbable anterior cervical graft containment plate. J Neurosurg (Spine 4) 97:440-446, 2002

2. Baisden J, Voo LM, Cusick JF, et al: Evaluation of cervical laminectomy and laminoplasty. A longitudinal study in the goat model. Spine 24:1283-1289, 1999

3. Brodke DS, Gollogly S, Mohr RA, et al: Dynamic cervical plates: biomechanical evaluation of load sharing and stiffness. Spine 26:1324-1329, 2001

4. Cain CC, Fraser RD: Bony and vascular anatomy of the normal cervical spine in the sheep. Spine 20:759-765, 1995

5. Cauthen JC, Kinard RE, Vogler JB, et al: Outcome analysis of noninstrumented anterior cervical discectomy and interbody fusion in 348 patients. Spine 23:188-192, 1998

6. Chen TY, Crawford NR, Sonntag VK, et al: Biomechanical effects of progressive anterior cervical decompression. Spine 26: 6-14, 2001

7. Cohen SR, Holmes RE: Internal Le Fort III distraction with biodegradable devices. J Craniofac Surg 12:264-272, 2001

8. Crawford NR, Brantley AG, Dickman CA, et al: An apparatus for applying pure nonconstraining moments to spine segments in vitro. Spine 20:2097-2100, 1995

9. Crawford NR, Dickman CA: Construction of local vertebral coordinate systems using a digitizing probe. Technical note. Spine 22:559-563, 1997

10. Crawford NR, Peles JD, Dickman CA: The spinal lax zone and neutral zone: measurement techniques and parameter comparisons. J Spinal Disord 11:416-429, 1998
11. Crawford NR, Yamaguchi GT, Dickman CA: A new technique for determining 3-D joint angles: the tilt/twist method. Clin Biomech 14:153-165, 1999

12. Grubb MR, Currier BL, Shih JS, et al: Biomechanical evaluation of anterior cervical spine stabilization. Spine 23:886-892, 1998

13. Hollinger JO, Battistone GC: Biodegradable bone repair materials. Synthetic polymers and ceramics. Clin Orthop 207: 290-305, 1986

14. Kandziora F, Pflugmacher R, Schafer J, et al: Biomechanical comparison of cervical spine interbody fusion cages. Spine 26: 1850-1857, 2001

15. Kandziora F, Pflugmacher R, Scholz M, et al: Comparison between sheep and human cervical spines: an anatomic, radiographic, bone mineral density, and biomechanical study. Spine 26:1028-1037, 2001

16. Kaptain GJ, Vincent DA, Laws ER Jr: Cranial base reconstruction after transsphenoidal surgery with bioabsorbable implants: technical note. Neurosurgery 48:232-234, 2001

17. Lee EJ, Hung YC, Lee MY, et al: Kinematics of cervical spine discectomy with and without bone grafting: quantitative evaluation of late fusion in a sheep model. Neurosurgery 44: 139-147, 1999

18. Lowery GL, McDonough RF: The significance of hardware failure in anterior cervical plate fixation. Patients with 2- to 7 year follow-up. Spine 23:181-187, 1998

19. Middleton JC, Tipton AJ: Synthetic biodegradable polymers as orthopedic devices. Biomaterials 21:2335-2346, 2000

20. Shono Y, McAfee PC, Cunningham BW, et al: A biomechanical analysis of decompression and reconstruction methods in the cervical spine. Emphasis on a carbon-fiber-composite cage. J Bone Joint Surg Am 75:1674-1684, 1993

21. Spivak JM, Chen D, Kummer FJ: The effect of locking fixation screws on the stability of anterior cervical plating. Spine 24: 334-338, 1999

22. Takahashi T, Tominaga T, Watabe N, et al: Use of porous hydroxyapatite graft containing recombinant human bone morphogenetic protein-2 for cervical fusion in a caprine model. J Neurosurg (Spine 4) 90:224-230, 1999

23. Ulrich C, Arand M, Nothwang J: Internal fixation on the lower cervical spine-biomechanics and clinical practice of procedures and implants. Eur Spine J 10:88-100, 2001

24. Vaccaro AR, Carrino JA, Venger BH, et al: Use of a bioabsorbable anterior cervical plate in the treatment of cervical degenerative and traumatic disc disruption. J Neurosurg (Spine 4) 97: 473-480, 2002

25. Wang JC, McDonough PW, Endow KK, et al: A comparison of fusion rates between single-level cervical corpectomy and twolevel discectomy and fusion. J Spinal Disord 14:222-225, 2001

26. Whitecloud TS III: Modern alternatives and techniques for onelevel discectomy and fusion. Clin Orthop 359:67-76, 1999

27. Wilke HJ, Kettler A, Claes LE: Are sheep spines a valid biomechanical model for human spines? Spine 22:2365-2374, 1997

28. Wilke HJ, Kettler A, Wenger KH, et al: Anatomy of the sheep spine and its comparison to the human spine. Anat Rec 247: $542-555,1997$

\section{Manuscript received January 15, 2004.}

Accepted in final form February 10, 2004.

This study was supported by Medtronic Sofamor Danek and MacroPore Biosurgery, Inc.

Address reprint requests to: Christopher P. Ames, M.D., Department of Neurological Surgery, University of California, San Francisco, 505 Parnassus Avenue M-779, San Francisco, California 94143-0112. email: amesc@ @eurosurg.ucsf.edu. 